\title{
The Wilson exact renormalization group equation and the anomalous dimension parameter
}

\author{
C. Bervillier* \\ Laboratoire de Mathématiques et Physique Théorique, UMR 7350 (CNRS), Fédération Denis Poisson, \\ Université François Rabelais, Parc de Grandmont, 37200 Tours, France
}

Received December 14, 2012, in final form March 8, 2013

The non-linear way the anomalous dimension parameter has been introduced in the historic first version of the exact renormalization group equation is compared to current practice. A simple expression for the exactly marginal redundant operator proceeds from this non-linearity, whereas in the linear case, first order differential equations must be solved to get it. The role of this operator in the construction of the flow equation is highlighted.

Key words: exact renormalization group equation, anomalous dimension

PACS: 05.10.Cc, 11.10.Gh, 64.60.ae

\section{Introduction}

The Wilson renormalization group (RG) ideas [1-3] have formalized and clarified the notions of scaling and of universality attached to critical behavior. As several modern subjects in physics, critical phenomena cannot be studied by pure perturbative methods. The development of nonperturbative methods is thus very important. As is well demonstrated by the work of M. Kozlovskii (see, e.g., [4-8]), the collective variables method [9], close to the early ideas of Kadanoff [10], is one of the RG-like nonperturbative methods allowing one to investigate the critical point, starting with a microscopic Hamiltonian - thus allowing one to calculate nonuniversal quantities such as the critical temperature [4]. The subject of the present article is another expression of the nonperturbative RG framework, called the exact RG equation (ERGE) - see in [1] section 11, equations (11.14, 11.15), and for reviews see, e.g., [11-19]. Though one could also calculate nonuniversal quantities with an ERGE (see, e.g., [20-22]), our purpose is presently limited to the close vicinity of a RG fixed point with a view to discuss the particular issue of the way one may account for the anomalous dimension of the field (the critical exponent $\eta$ ) within an ERGE.

Among an infinite variety of equivalent ERGE, only those based on an effective cutoff function associated with a bilinear kinetic term are currently used [23]. Despite this limited number of usual variants (a priori close to each other), they show differences sufficient to make it sometimes difficult to clearly display (or even understand) their relationships. Of course, several authors have addressed this issue in general [24-30] but, in contrast to [31-34], the anomalous dimension parameter $\eta$ was not included in their considerations. For example, it is known that the Wilson historic first version [1] is made equivalent to the Polchinski version [35], provided there is a choice of an exponential cutoff function and after a specific field redefinition [28, 31], but the parameter $\eta$ was not explicitly considered in this relation except in [31].

Recently Osborn and Twigg [33] and Rosten [32, 34] have independently established the relation between a version of the Polchinski ERGE — "modified" to include the anomalous dimension [36] — and a "fixed-point equation" for the effective average action [28]. This relation appears to be rather complicated: it "involves solving some first order differential equations" [33]. O’Dwyer and Osborn [37] had

\footnotetext{
*E-mail: claude.bervillier@lmpt.univ-tours.fr
} 
previously encountered similar first order differential equations in the process of constructing the (exactly) marginal, redundant "operator' $11 \mathcal{O}$ (EMRO) associated with the change of normalization of the field by a constant factor but they did not discuss the effect of the way $\eta$ had been accounted for in the ERGE on this complexity. Yet, it is a well known fact that the EMRO of the Wilson version [1] takes on a simple form - e.g., see equation (24) of [12] or equation (2.35) of [38]. The object of the present article is to compare the main ways of introducing $\eta$ in an ERGE encountered in the literature. We show, in particular, that the simplicity of the form of the EMRO is maintained in the Wilson version of the ERGE extended to an arbitrary cutoff function [31]. The role of the EMRO in the construction of an ERGE is also highlighted. A larger discussion is left to an upcoming detailed publication [39].

\section{The RG steps}

\subsection{Reminder}

Basically, a RG transformation of a cutoffed action $S[\phi, \ell]$ of a scalar field $\phi$ involves three steps. Having chosen a fixed arbitrary momentum scale of reference $\Lambda_{0}$, and having defined the RG-scale parameter $\ell=\Lambda / \Lambda_{0} \leqslant 1$, these three steps are:

Step 1: an integration of the high momentum components of the field generating an effective action with a reduced cutoff $\Lambda^{\prime}=(1-d \ell / \ell) \Lambda$.

Step 2: a rescaling of the momenta back to the initial value of the cutoff: $|q| \rightarrow(1-d \ell / \ell)|q|$. This step is accounted for by a simple dimensional analysis such as defining a dimensionless momentum as $\tilde{\mathbf{q}}=\mathbf{q} / \Lambda$ and a dimensionless field $\tilde{\phi}_{\tilde{q}}=\Lambda^{-\bar{d}_{\phi}} \phi_{q}$ [see also equation (7)].

Step 3: a renormalization of the field $\tilde{\phi}_{\tilde{q}} \longrightarrow \zeta(\ell-d \ell) \tilde{\phi}_{\tilde{q}}$, with $\zeta(\ell)=\ell^{\varpi}$ and $\varpi$ related to $\eta$, e.g., see equation (19). This step is required to keep constant (i.e., independent of $\ell$ ) one term of $S$ in order to set up the system of scale of reference.

Having defined the RG-time $t$ as:

$$
t=-\ln \ell=-\ln \left(\frac{\Lambda}{\Lambda_{0}}\right)
$$

the combined effect of the above three steps on the action $S$ may be expressed as:

$$
\dot{S}\left[\tilde{\phi}, e^{-t}\right]=\mathscr{G}_{\text {tra }}(S)+\mathscr{G}_{\text {dil }}\left(S, d_{\phi}\right)+\mathscr{G}_{\text {ren }}(S),
$$

in which:

$$
\begin{aligned}
\dot{S}\left[\tilde{\phi}, \mathrm{e}^{-t}\right] & \left.\equiv \frac{\mathrm{d}}{\mathrm{d} t} S\left[\tilde{\phi}, \mathrm{e}^{-t}\right]\right|_{\tilde{\phi}}, \\
\mathscr{G}_{\text {dil }}\left(S, d_{\phi}\right) & =\int_{\tilde{p}}\left(\tilde{\mathbf{p}} \cdot \frac{\partial \tilde{\phi}_{\tilde{p}}}{\partial \tilde{\mathbf{p}}}-\bar{d}_{\phi} \tilde{\phi}_{\tilde{p}}\right) \frac{\delta S}{\delta \tilde{\phi}_{\tilde{p}}} .
\end{aligned}
$$

The expression of $\mathscr{G}_{\text {tra }}(S)$ in (2), depends on the way the above step 1 is realized. In any case, for a complete action $S$, it corresponds to a (quasi local) field redefinition which leaves the partition function unchanged and, consequently, must have the form [2, 3]:

$$
\begin{aligned}
& \mathscr{G}_{\operatorname{tra}}(S)=\Xi[S, \Psi], \\
& \Xi[S, \Psi]=\int_{\tilde{q}}\left(\Psi_{\tilde{q}} \frac{\delta S}{\delta \tilde{\phi}_{\tilde{q}}}-\frac{\delta \Psi_{\tilde{q}}}{\delta \tilde{\phi}_{\tilde{q}}}\right),
\end{aligned}
$$

in which $\Psi_{\tilde{q}}$ is some functional of $\tilde{\phi}$.

\footnotetext{
${ }^{1}$ Actually $\mathscr{O}$ is not an operator but represents a direction in the space of actions $\{S\}$.
} 
As for the expression of $\mathscr{G}_{\text {ren }}(S)$, it is the object of the present article to discuss its various forms encountered in the literature. It is related to the expression of $\bar{d}_{\phi}$ in (4); if $d_{\phi}$ is the dimension of $\phi(x)$, $\bar{d}_{\phi}$ is that of its Fourier transformed $\phi_{q}$, if $D$ is the spatial dimension, they are related through:

$$
\bar{d}_{\phi}=d_{\phi}-D .
$$

In the following we use two kinds of $d_{\phi}$ : a "classical” family $d_{\phi}^{(c)}$ :

$$
d_{\phi}^{(c)}=\frac{D}{2}-n_{0}
$$

in which ${ }^{2} n_{0}$ is related to the behavior of the cutoff function at small momenta [see equations (16, 17) where $n_{0}=0$ or $\left.n_{0}=1\right]$, and the fixed-point-value dimension:

$$
d_{\phi}^{(a)}=\frac{D-2+\eta}{2}
$$

in which $\eta$ is the usual critical exponent.

\subsection{Main ways of introducing $\eta$}

Though it gave the name to the theory, step 3 is currently skipped in the modern constructions of an ERGE. A perfect example of this fact is the original Polchinski ERGE [35] a version of which — including $\eta$ as proposed in [36] — has been called the "modified" Polchinski version [32, 34] or the Polchinski version "extended to include the parameter $\eta$ " [33]. In modern approaches, the renormalization step 3 is currently avoided and $\eta$ is merely included in the rescaling step 2 via some ad hoc "anomalous" dimensional analysis [28, 40]. There is nothing wrong with such a procedure: the large freedom that is offered in the construction of an ERGE makes it well acceptable. It remains no less true that it is fair to ask whether or not there are important consequences of doing this or that.

Essentially, two ways of introducing $\eta$ are encountered in the literature:

1. via the explicit renormalisation step 3 associated with $\mathscr{G}_{\mathrm{ren}}(S)$. In that case the dimension $d_{\phi}$ in $\mathscr{G}_{\text {dil }}\left(S, d_{\phi}\right)$ is classical, given by (8). This is the original and most general procedure but it is not currently used.

2. via some "anomalous" dimensional analysis as proposed, e.g., by Morris [28]. In that case $\mathscr{G}_{\text {ren }}(S) \equiv$ 0 and $d_{\phi}$ is given by (9). In fact, this procedure, or some equivalent variant, is generally used in the current versions of the ERGE (see also, e.g., Berges et al [13], Ball et al [36] etc. ...). It is a correct procedure in case of the proximity of a fixed point.

In addition, one may observe that the effective contribution of $\eta$ within the ERGE is either purely linear or not (w.r.t. S). For example, the procedure of Ball et al [36] is linear whereas the Wilson [1], Morris [28] and Wetterich [13] procedures are non-linear. These differences, although well allowed, have some consequences in the relations between the main versions of the ERGE. In the following sections we examine the non-linear Wilson procedure in greater detail than previously done in [31] and compare it with the linear and non-linear procedures of, respectively, Ball et al [36] and Morris [28].

\section{The extended Wilson ERGE}

\subsection{Presentation}

Let us consider the extended Wilson ERGE (i.e., the flow equation of $S$ with an arbitrary cutoff function), as obtained in [31]. For convenience, we adopt the notations of Osborn and Twigg [33] and write

\footnotetext{
${ }^{2}$ The value of $d_{\phi}^{(\mathrm{c})}$ depends on which term is chosen to set the standard of the scale of reference. Usually one refers to the kinetic term and $d_{\phi}^{(\mathrm{c})}=\frac{D-2}{2}\left(n_{0}=1\right)$. Wilson has chosen a pure quadratic term and, for him $d_{\phi}^{(\mathrm{c})}=\frac{D}{2}\left(n_{0}=0\right)$.
} 
the flow equation under the form of (2) with (notice the change in $\mathscr{G}_{\text {dil }}$ ):

$$
\begin{aligned}
\mathscr{G}_{\text {tra }}(S) & =\int_{\tilde{q}}\left[G\left(\tilde{q}^{2}\right)\left(\frac{\delta^{2} S}{\delta \tilde{\phi}_{\tilde{q}} \delta \tilde{\phi}_{-\tilde{q}}}-\frac{\delta S}{\delta \tilde{\phi}_{\tilde{q}}} \frac{\delta S}{\delta \tilde{\phi}_{-\tilde{q}}}\right)+H\left(\tilde{q}^{2}\right) \tilde{\phi}_{\tilde{q}} \frac{\delta S}{\delta \tilde{\phi}_{\tilde{q}}}\right], \\
\mathscr{G}_{\text {ren }}(S) & =\Subset \mathscr{O}(S, \tilde{P}), \\
\mathscr{G}_{\text {dil }}\left(S, d_{\phi}\right) & =\mathscr{G}_{\text {dil }}\left(S, d_{\phi}^{(c)}\right),
\end{aligned}
$$

where:

$$
\begin{aligned}
& \mathscr{O}(S, \tilde{P})=\int_{\tilde{q}}\left[\tilde{P}\left(\tilde{q}^{2}\right)\left(\frac{\delta^{2} S}{\delta \tilde{\phi}_{\tilde{q}} \delta \tilde{\phi}_{-\tilde{q}}}-\frac{\delta S}{\delta \tilde{\phi}_{\tilde{q}}} \frac{\delta S}{\delta \tilde{\phi}_{-\tilde{q}}}\right)+\tilde{\phi}_{\tilde{q}} \frac{\delta S}{\delta \tilde{\phi}_{\tilde{q}}}\right], \\
& G\left(\tilde{q}^{2}\right)=-\tilde{q}^{2} \tilde{P}\left(\tilde{q}^{2}\right) \frac{K^{\prime}\left(\tilde{q}^{2}\right)}{K\left(\tilde{q}^{2}\right)}, \\
& H\left(\tilde{q}^{2}\right)=-2 \tilde{q}^{2} \frac{K^{\prime}\left(\tilde{q}^{2}\right)}{K\left(\tilde{q}^{2}\right)} .
\end{aligned}
$$

Compared to [31], the cutoff function $P\left(q^{2}, \Lambda\right)$ has been given the (regular) dimension $\operatorname{dim}[P]=-2 n_{0}$ so that:

$$
\begin{aligned}
P\left(q^{2}, \ell \Lambda_{0}\right) & =\Lambda_{0}^{-2 n_{0}} \ell^{2 \omega-2 n_{0}} \tilde{P}\left(\tilde{q}^{2}\right), \\
\tilde{P}\left(\tilde{q}^{2}\right) & =\frac{K\left(\tilde{q}^{2}\right)}{\left(\tilde{q}^{2}\right)^{n_{0}}},
\end{aligned}
$$

where $K$ is dimensionless - this is to permit comparison with the current uses where $n_{0}=1$ whereas $n_{0}=0$ in [31].

It is useful to recall the following points:

- the general form of an ERGE involves an additive field independent term which will be sytematically neglected in the following;

- the establishment of the extended Wilson ERGE is based on the relation of the complete action $S$ to a partial action $S_{\text {int }}$ by extracting a quadratic form involving the arbitrary cutoff function $P$ :

$$
S[\phi]=\frac{1}{2} \int_{q} \phi_{q} P^{-1}\left(q^{2}, \ell \Lambda_{0}\right) \phi_{-q}+S_{\text {int }}[\phi] ;
$$

- in its original version 35] Polchinski expresses the flow of $S_{\text {int }}$ under a change of $\ell-$ instead of $S$ for the Wilson ERGE;

- the factorized $\ell^{2 \oplus}$-term in (16) was not part of Polchinski's assumptions. This kind of $\ell$-dependency in front of the cutoff function is a convenient artefact to introduce $\eta$ non-linearly in the ERGE. This is not unusual since Morris [28] already used it when he gave the cutoff function an anomalous dimension (see section 4.2). We shall show that the recourse to the EMRO enables us to get rid of this artefact;

- in order to specify the nature of the renormalization step, one must make reference to the right power law behavior at large distances of the critical two point correlation function and this implies that [31]:

$$
\omega=1-n_{0}-\frac{\eta}{2}
$$

[In the case of anomalous dimensional analysis, this step is not required since the field has a priori been given the "right" fixed point dimension $d_{\phi}^{(a)}$ defined by [9]]; 
- it is easy to verify that for $n_{0}=0$ and $\omega$ given by (19), (10)-17) gives the historic first version of the ERGE [1] with the choice $\tilde{P}\left(\tilde{q}^{2}\right)=\mathrm{e}^{-2 \tilde{q}^{2}}$, and the redefinition of the field [31]:

$$
\tilde{\phi}_{\tilde{q}} \rightarrow \sqrt{\tilde{P}\left(\tilde{q}^{2}\right)} \tilde{\phi}_{\tilde{q}}
$$

- the writing of equations (10)-15) suggests that $\mathscr{O}(S, \tilde{P})$ plays the role of the redundant "operator" $\mathscr{O}(S, 1)$ which is associated with an infinitesimal renormalization of the field by a constant factor [38, 41]. It is, actually, via $\mathscr{O}(S, 1)$ that $\eta$ was introduced in the historic first version 3 .

Instead of assuming the presence of the factor $\ell^{2 \omega}$ in $(16)$, we could as well have implemented step 3 using $\mathscr{O}(S, 1)$ in place of $\mathscr{O}(S, \tilde{P})$ in $(11)$, but then this would have destroyed the currently admitted equivalence [23, 28] [under the change [20)] between Polchinski's and Wilson's ERGE. This is merely because, given a fixed point $S^{*}$ [characterized by $\dot{S}^{*}=0$ ], $\mathscr{O}\left(S^{*}, 1\right)$ is not an EMRO for the extended Wilson ERGE whereas $\mathscr{O}\left(S^{*}, \tilde{P}\right)$ is. It is the object of the next section to discuss this issue.

\subsection{The exactly marginal redundant "operator"}

In this section, we apply the procedure described in appendix D of [37] to the complete action $S$ (see also [19]) and we demonstrate that $\mathscr{O}(S, \tilde{P})$ defined by (13) with $S=S^{*}$, corresponds to an EMRO for the ERGE given by (2), (10)-(12), and that it may be used to implement the RG step 3.

It is easy to see that $\mathscr{O}(S, \tilde{P})$ is a redundant "operator" since it may be written under the form of the r.h.s. of (6) with:

$$
\Psi_{\tilde{q}}^{(\tilde{P})}=\tilde{\phi}_{\tilde{q}}-\tilde{P}\left(\tilde{q}^{2}\right) \frac{\delta S}{\delta \tilde{\phi}_{-\tilde{q}}}
$$

We then proceed in two stages, showing successively that:

Stage 1: whatever the functions $G\left(\tilde{q}^{2}\right)$ and $H\left(\tilde{q}^{2}\right)$ :

(a) there exists a function $B\left(q^{2}\right)$ - solution of a first order differential equation - such that $\mathscr{O}\left(S^{*}, B\right)$ is exactly marginal for the ERGE without renormalization (i.e., with $\mathscr{G}_{\text {ren }}(S) \equiv 0$ and an arbitrary dimension parameter $d_{\phi}$ ),

(b) $\mathscr{O}\left(S^{*}, B\right)$ is again exactly marginal for the ERGE translated by $\alpha \mathscr{O}(S, B)$ [i.e., with $\left.\mathscr{G}_{\text {ren }}(S)=\alpha \mathscr{O}(S, B)\right]$ with $\alpha$ an arbitrary constant.

Stage 2: $B\left(q^{2}\right)$ identifies with $\tilde{P}\left(\tilde{q}^{2}\right)$ in the case of (14), 15).

Let us consider the ERGE linearized about a fixed point $S^{*}$ and its associated eigenvalue equation for some eigenfunctional $\Theta_{i}[\tilde{\phi}]$ :

$$
\hat{\mathscr{D}} \Theta_{i}=\lambda_{i} \Theta_{i}
$$

For the ERGE given by (2), (10)-(12), without the renormalisation part $\mathscr{G}_{\text {ren }}(S)$ and for arbitrary $d_{\phi}$,

\footnotetext{
${ }^{3}$ Via a scale dependent factor $\Phi(t)$ in front of $\mathscr{O}(S, 1)$ instead of a constant $\Phi$ as in [1], $\varrho(t)$ coincides with $\varrho$ in the vicinity of a fixed point.
} 
we have:

$$
\begin{aligned}
\hat{\mathscr{D}} & =\hat{\mathscr{D}}_{1}+\hat{\mathscr{D}}_{2}+\hat{\mathscr{D}}_{3}+\hat{\mathscr{D}}_{4}, \\
\hat{\mathscr{D}}_{1} & =\int_{\tilde{p}}\left[\tilde{\mathbf{p}} \cdot \frac{\partial \tilde{\phi}_{\tilde{p}}}{\partial \tilde{\mathbf{p}}}-\bar{d}_{\phi} \tilde{\phi}_{\tilde{p}}\right] \frac{\delta}{\delta \tilde{\phi}_{\tilde{p}}}, \\
\hat{\mathscr{D}}_{2} & =\int_{\tilde{p}} G\left(\tilde{p}^{2}\right) \frac{\delta^{2}}{\delta \tilde{\phi}_{\tilde{p}} \delta \tilde{\phi}_{-\tilde{p}}}, \\
\hat{\mathscr{D}}_{3} & =\int_{\tilde{p}} H\left(\tilde{p}^{2}\right) \tilde{\phi}_{\tilde{p}} \frac{\delta}{\delta \tilde{\phi}_{\tilde{p}}}, \\
\hat{\mathscr{D}}_{4} & =-2 \int_{\tilde{p}} G\left(\tilde{p}^{2}\right) \frac{\delta S^{*}}{\delta \tilde{\phi}_{\tilde{p}}} \frac{\delta}{\delta \tilde{\phi}_{-\tilde{p}}} .
\end{aligned}
$$

Considering a general redundant "operator" $\Xi[S, \Psi]$ as defined by (6), it is not very complicated to verify that the general property [2, 3] of the redundant "operators" to form a closed subspace under the flow in the vicinity of a fixed-point takes on the form:

$$
\hat{\mathscr{D}} \Xi\left[S^{*}, \Psi\right]=\Xi\left[S^{*}, \hat{\mathscr{D}}_{t} \Psi\right],
$$

with:

$$
\hat{\mathscr{D}}_{t} \Psi_{\tilde{q}}[\tilde{\phi}]=\left(\hat{\mathscr{D}}+\bar{d}_{\phi}-G\left(\tilde{q}^{2}\right)-\tilde{\mathbf{q}} \cdot \frac{\partial}{\partial \tilde{\mathbf{q}}}\right) \Psi_{\tilde{q}}[\tilde{\phi}] .
$$

Following the procedure of [37], we try to build up the EMRO [i.e., a solution $\Theta_{0}$ of [22) with $\lambda_{0}=0$ ] from equations (23), (24) with a function $\Psi_{\tilde{q}}^{(B)}[\tilde{\phi}]$ similar to (21) and find that $B(x)$ must be the solution of the following first order differential equation (with initial value):

$$
\begin{aligned}
& G(x)-\varpi_{g} B(x)-H(x) B(x)-x B^{\prime}(x)=0, \\
& B(0)=\frac{G(0)}{\varpi_{g}},
\end{aligned}
$$

in which:

$$
\varrho_{g}=-\frac{d_{\phi}+\bar{d}_{\phi}}{2}
$$

Assuming an explicit solution of $B(x)$ [see equations [31), (32)] this closes stage 1a.

The quantity $\mathscr{O}(S, B)$, similar to (13), is thus redundant and exactly marginal at the fixed point $S^{*}$. Let us consider a new ERGE with:

$$
\mathscr{G}_{\text {ren }}(S)=\alpha \mathscr{O}(S, B) .
$$

We easily see that this modification amounts to merely having performed the following translations in the flow equation:

$$
\begin{aligned}
G(x) & \longrightarrow G(x)+\alpha B, \\
\omega_{g} & \longrightarrow \omega_{g}+\alpha,
\end{aligned}
$$

which keep equation (25) unchanged. Consequently, stage $1 \mathrm{~b}$ is also verified. It is clear that this would not be the case using $\mathscr{O}(S, 1)$ in $(28)$.

The solution of the differential equations (25), (26) is:

$$
\begin{aligned}
B(x) & =\left(\frac{1}{x}\right)^{\Phi_{g}} \frac{1}{C_{0}(x)} \int_{0}^{x} u^{\Phi_{g}-1} G(u) C_{0}(u) \mathrm{d} u, & \text { if } & \emptyset_{g} \geqslant 0, \\
C_{0}(x) & =\exp \left\{\int_{0}^{x} \frac{H(u)}{u} \mathrm{~d} u\right\}, & \text { if } & H(0)=0 .
\end{aligned}
$$


Using an integration by parts, one may verify that $B(x)$, as given by (31), satisfies the initial condition (26), provided that $\varpi_{g} \geqslant 0$.

In the case where the functions $G$ and $H$ are defined by (14), (15), equation (31) simplifies to give:

$$
B(x) \equiv \tilde{P}(x)
$$

so that with $\Phi_{g}=\emptyset$, as given by $[19$, the quantity $\varrho \mathscr{O}(S, \tilde{P})$ corresponds to the effective non-linear realization of the field renormalization adapted to the ERGE under consideration (QED).

In order to discuss the linear implementation of $\eta$, it is useful to first consider the relation back to the original Polchinski version of the ERGE.

\section{From Wilson to Polchinski}

In this section we scrupulously look at the relation between the Wilson and the Polchinski ERGE considering successively the linear (Ball et al [36]) and non-linear (Wilson [1, 31], Morris [28]) ways of introducing $\eta$.

The Polchinski equation for $S_{\text {int }}[35$ ] is originally limited to the implementation of the RG step 1 via the variation of $S_{\text {int }}$ under the change of $\Lambda$ introduced by a bilinear term in $S$ [as shown in (18)]. It reads:

$$
\left.\ell \frac{\partial}{\partial \ell} S_{\mathrm{int}}\right|_{\phi}=-\left.\frac{1}{2} \int_{q} \ell \frac{\partial}{\partial \ell} P\left(q^{2}, \ell \Lambda_{0}\right)\right|_{q}\left[\frac{\delta^{2} S_{\mathrm{int}}}{\delta \phi_{q} \delta \phi_{-q}}-\frac{\delta S_{\mathrm{int}}}{\delta \phi_{q}} \frac{\delta S_{\mathrm{int}}}{\delta \phi_{-q}}\right]
$$

From this incomplete expression of the RG flow, the shift to $S$ is easily implemented via the following functional derivatives of (18):

$$
\begin{aligned}
\frac{\delta S_{\mathrm{int}}}{\delta \phi_{q}} & =\frac{\delta S}{\delta \phi_{q}}-P^{-1} \phi_{-q}, \\
\frac{\delta^{2} S_{\mathrm{int}}}{\delta \phi_{q} \delta \phi_{-q}} & =\frac{\delta^{2} S}{\delta \phi_{q} \delta \phi_{-q}}-P^{-1}
\end{aligned}
$$

from which we get (up to an additive field-independent term):

$$
\left.\ell \frac{\partial}{\partial \ell} S[\phi]\right|_{\phi}=-\frac{1}{2}\left\{\left.\int_{q} \ell \frac{\partial}{\partial \ell} P\left(q^{2}, \ell \Lambda_{0}\right)\right|_{q}\left[\frac{\delta^{2} S}{\delta \phi_{q} \delta \phi_{-q}}-\frac{\delta S}{\delta \phi_{q}} \frac{\delta S}{\delta \phi_{-q}}+2 P^{-1}\left(q^{2}, \ell \Lambda_{0}\right) \phi_{q} \frac{\delta S}{\delta \phi_{q}}\right]\right\} .
$$

Then, depending on the procedure chosen for introducing $\eta$, the implementation of the RG steps 2 and 3 may be easier to get a hand on according to whether one considers (34) or (37). In the linear case of Ball et al [36] this does not matter, however.

\subsection{Linear introduction of $\boldsymbol{\eta}$}

The cumulative account from $\Lambda_{0}$ to $\Lambda=\ell \Lambda_{0}$ which has been conveniently included as an explicit $\ell$-factor within the cutoff function [as displayed in [16]] is not necessary to the construction of an ERGE . Actually, only the reduction of the degrees of freedom in the infinitesimal range $\left[\Lambda,\left(1-\frac{d \ell}{\ell}\right) \Lambda\right]$ is obligatory. In other terms: the renormalization step 3 may be implemented linearly only. This is the procedure adopted by Ball et al [36] when they introduced $\eta$. However, there is a supplementary freedom.

The role of the renormalization step 3 is to compensate the modification of one term of $S$ induced by the implementation of the RG step 1. Instead of explicitly renormalizing the field, one may as well view this modification as an integral part of $\phi$ so that it may be absorbed in the rescaling step 2 by assuming that $\phi$ has the (anomalous) dimension (9) - notice that this implies $n_{0}=1$. Ball et al [36] have chosen that possibility. For both $S$ and $S_{\text {int }}$, RG steps 2 and 3 are then implemented by a mere dimensional analysis that readily provides the final flow equations. In terms of $t$ defined in (1) we get: 
- the flow equation for $S$ under the form (2) with $\mathscr{G}_{\text {ren }}(S)=0, \mathscr{G}_{\text {dil }}\left(S, d_{\phi}\right)=\mathscr{G}_{\text {dil }}\left(S, d_{\phi}^{(a)}\right)$ and $\mathscr{G}_{\text {tra }}(S)$ given by (10), 14), 15);

- the flow equation for $S_{\text {int }}$ as:

$$
\begin{aligned}
\dot{S}_{\text {int }} & =\mathscr{G}_{\text {tra }}\left(S_{\text {int }}\right)+\mathscr{G}_{\text {dil }}\left(S_{\text {int }}, d_{\phi}^{(a)}\right) \\
\mathscr{G}_{\text {tra }}\left(S_{\text {int }}\right) & =\int_{\tilde{q}}\left[G\left(\tilde{q}^{2}\right)\left(\frac{\delta^{2} S_{\text {int }}}{\delta \tilde{\phi}_{\tilde{q}} \delta \tilde{\phi}_{-\tilde{q}}}-\frac{\delta S_{\text {int }}}{\delta \tilde{\phi}_{\tilde{q}}} \frac{\delta S_{\text {int }}}{\delta \tilde{\phi}_{-\tilde{q}}}\right)\right]
\end{aligned}
$$

with $G\left(\tilde{q}^{2}\right)$ given by (14).

However, since the anomalous dimension attributed to $\phi$ is not compensated by an anomalous dimension of the cutoff function in the bilinear term of $S$, the relation between the two flow equations is altered and we have:

$$
\dot{S}=\dot{S}_{\mathrm{int}}+\omega \int_{\tilde{q}} \tilde{\phi}_{\tilde{q}} \tilde{P}^{-1}\left(\tilde{q}^{2}\right) \tilde{\phi}_{-\tilde{q}}
$$

with $\varrho$ given by (19) in which $n_{0}=1$. This implies that the two flow equations do not have equivalent fixed points.

Equation (40) has induced the notion of "modified" Polchinski flow equation [32-34] which refers to the flow equation for $S$ (expressed in terms of $S_{\text {int }}$ ) rather than to equations [38, (39) as it would be normally. It is worth to underline, however, that the latter flow equation for $S_{\text {int }}$ is perfectly valid and may be studied for its own sake.

Another consequence of the linear introduction of $\eta$ is the complicated expression of the EMRO. Indeed, the linear introduction of $\eta$ is not compatible with the translation (29), (30) and the solution of (25), (26) must actually be considered with $\Phi_{g}=1-\eta / 2$ so that, for the choice (14), (15) and with $\tilde{P}\left(\tilde{q}^{2}\right)$ given by (16) in which $\varrho$ is formally set equal to 0 , we get, provided $\eta<2$ :

$$
B(x)=\left(\frac{1}{x}\right)^{1-\eta / 2}[K(x)]^{2} \int_{0}^{x} u^{-\eta / 2} \frac{K^{\prime}(u)}{[K(u)]^{2}} \mathrm{~d} u,
$$

which cannot be reduced to the simple form of [33), for arbitrary $K$. The result (41) is equivalent to that obtained in appendix D of [37] for the EMRO constructed in terms of $S_{\text {int. }}$.

\subsection{Non-linear introduction of $\boldsymbol{\eta}$}

In this section we compare two non-linear ways of introducing $\eta$ : the Morris version [28] which is based on an "anomalous" dimensional analysis and the Wilson version extended to an arbitrary cutoff described in section 3 . We show that the two versions are formally very close to each other but yield different RG flow equations.

In [28], Morris has a priori given the field $\phi$ the dimension $d_{\phi}^{(a)}$ [given by (9)] with an anomalous part that is compensated by a cutoff function anomalously dimensioned. Applied to our present matter, the direct consequence (the easiest to grasp) of the Morris procedure is the modification of (40) into:

$$
\dot{S}=\dot{S}_{\text {int }}
$$

that expresses the interesting property of the flow equations for $S$ and $S_{\text {int }}$ to have equivalent fixed points. It is important to notice that this property is also true with the Wilson version extended to an arbitrary cutoff function since, by construction, the renormalization of the field exactly compensates the extra $\ell$ dependency of the cutoff function displayed in (16).

To get the Morris version of the flow equations for $S$ and $S_{\text {int }}$, one must first come back to the derivation of the Polchinski-like equation (34) where the derivative w.r.t. $\ell$ is performed at a fixed dimensioned field (RG step 1). This time, $P\left(q^{2}, \ell \Lambda_{0}\right)$ is not given by (16) but, for dimensional reason, by 


$$
P\left(q^{2}, \ell \Lambda_{0}\right)=\Lambda_{0}^{-2 \oplus} \ell^{-2 \Phi} \tilde{P}\left(\tilde{q}^{2}\right),
$$

with $\varrho$ given by (19) in which $n_{0}$ is equal to zero to avoid singularities at $|q|=0$. Consequently, after the rescaling step 2, it comes a flow equation for $S_{\text {int }}$ of the form (38), (39) but with an effective $G\left(\tilde{q}^{2}\right)$ translated by the constant term $-\oplus$ compared to (14) so that this flow equation may finally be written under the form:

$$
\dot{S}_{\text {int }}=\mathscr{G}_{\text {tra }}\left(S_{\text {int }}\right)+\mathscr{G}_{\text {dil }}\left(S_{\text {int }}, d_{\phi}^{(\mathrm{c})}\right)-\varpi \mathscr{O}\left(S_{\text {int }}, \tilde{P}\right),
$$

in which $\mathscr{O}\left(S_{\text {int }}, \tilde{P}\right)$ is the expression in terms of $S_{\text {int }}$ of the redundant "operator" obtained from (13) using (35), (36) (up to a field independent term):

$$
\mathscr{O}\left(S_{\mathrm{int}}, \tilde{P}\right)=\int_{\tilde{q}}\left[\tilde{P}\left(\tilde{q}^{2}\right)\left(\frac{\delta^{2} S_{\mathrm{int}}}{\delta \tilde{\phi}_{\tilde{q}} \delta \tilde{\phi}_{-\tilde{q}}}-\frac{\delta S_{\mathrm{int}}}{\delta \tilde{\phi}_{\tilde{q}}} \frac{\delta S_{\mathrm{int}}}{\delta \tilde{\phi}_{-\tilde{q}}}\right)-\tilde{\phi}_{\tilde{q}} \frac{\delta S_{\mathrm{int}}}{\delta \tilde{\phi}_{\tilde{q}}}\right] .
$$

The Morris-like flow equation for $S$ is then readily obtained under the form:

$$
\dot{S}=\mathscr{G}_{\text {tra }}(S)+\mathscr{G}_{\text {dil }}\left(S, d_{\phi}^{(\mathrm{c})}\right)-\mathscr{G}_{\text {ren }}(S),
$$

in which the three terms are defined by equations (10)-12). Notice the negative sign in front of $\mathscr{G}_{\text {ren }}(S)$ which is opposite to that of Wilson as described in section (3) - one would observe the same change of sign in front of $\varrho$ in (44) compared to its Wilson-like version. This difference is due to the fact that some aspects have formally been reversed. On the one hand, (Wilson) one lets the coefficients of $S$ vary under the change of scale and then renormalizes the field. On the other hand, the "renormalization step" is anticipated and included within the cutoff function because the (useful) variation of the coefficients of $S$ has been arbitrarily incorporated in the scaling property of the field. Notice that in both cases the procedure amounts to keeping the same term of the action constant.

The two versions provide well allowed forms of ERGE, but it is worth underlining the unusual expression of (46) that may have consequences in practical calculations. The discussion of that issue - as well as of the consequences of other considerations presented in this article - is left to another publication [39].

\section{Summary and conclusion}

Three different ways of introducing the anomalous dimension parameter $\eta$ in an ERGE have been considered explicitly. The non-linear procedure of the historic first version [1] (extended to an arbitrary cutoff function in [31]) has been compared to the linear and non-linear versions associated with the "anomalous" dimensional analysis procedure of respectively the "modified" Polchinski version [32-34, 36] and the Morris version [28]. Their differences in essence have been emphasized, as well as the reasons why the non-linear versions should provide simpler calculational frameworks. The role of the exactly marginal redundant operator in the construction of an ERGE has also been underlined.

\section{Acknowledgements}

It is with great pleasure that I dedicate this article to M. Kozlovskii on his sixtieth anniversary and in remembrance of the times when we organized some symposium in the early 1990s.

I thank H. Osborn for useful remarks.

\section{References}

1. Wilson K.G., Kogut J., Phys. Rep., 1974, 12C, 75; doi 10.1016/0370-1573(74)90023-4

2. Wegner F.J., J. Phys. C: Solid State Phys., 1974, 7, 2098; doi 10.1088/0022-3719/7/12/004

3. Wegner F.J., In: Phase Transitions and Critical Phenomena, Vol. VI, Domb C., Green M.S. (Eds.), Academic Press, New York, 1976, p. 7. 
4. Kozlovskii M.P., Pylyuk I.V., Usatenko Z.E., Phys. Stat. Sol. B, 1996, 197, 465; doi 10.1002/pssb.2221970221

5. Kozlovskii M.P., Patsahan O.V., Condens. Matter Phys., 2000, 3, 607.

6. Kozlovskii M.P., Pylyuk I.V., Prytula O.O., Phys. Rev. B, 2006, 73, 174406; doi 10.1103/PhysRevB.73.174406

7. Kozak P.R., Kozlovskii M.P., Usatenko Z.E., J. Phys. A: Math. Theor., 2010, 43, 495001; doi 10.1088/1751-8113/43/49/495001

8. Kozlovskii M.P., Romanik R.V., Condens. Matter Phys., 2011, 14, 43002; doi 10.5488/CMP.14.43002

9. Yukhnovskii I.R., Phase Transitions of the Second Order. Collective Variables Method, World Scientific, Singapore, 1987.

10. Kadanoff L.P., Physics, 1966, 2, 263.

11. Aoki K.-I., Int. J. Mod. Phys. B, 2000, 14, 1249; doi 10.1142/S0217979200000923

12. Bagnuls C., Bervillier C., Phys. Rep., 2001, 348, 91; doi 10.1016/S0370-1573(00)00137-X.

13. Berges J., Tetradis N., Wetterich C., Phys. Rep., 2002, 363, 223; doi 10.1016/S0370-1573(01)00098-9

14. Polonyi J., Cent. Eur. J. Phys., 2003, 1, 1; doi 10.2478/BF02475552

15. Delamotte B., Mouhanna D., Tissier M., Phys. Rev. B, 2004, 69, 134413; doi 10.1103/PhysRevB.69.134413

16. Pawlowski J.M., Ann. Phys., 2007, 322, 2831; doi 10.1016/j.aop.2007.01.007

17. Delamotte B., In: Order, Disorder and Criticality. Advanced Problems of Phase Transition Theory, Vol. 2, Holovatch Yu. (Ed.), World Scientific, Singapore, 2007, p. 1.

18. Kopietz P., Bartosch L., Schütz F., Lecture Notes in Physics, 2010, 798; doi 10.1007/978-3-642-05094-7

19. Rosten O.J., Phys. Rep., 2012, 511, 177; doi 10.1016/j.physrep.2011.12.003

20. Seide S., Wetterich C., Nucl. Phys. B, 1999, 562, 524; doi 10.1016/S0550-3213(99)00545-3

21. Tissier M., Delamotte B., Mouhanna D., Phys. Rev. Lett., 2000, 84, 5208; doi 10.1103/PhysRevLett.84.5208

22. Machado T., Dupuis N., Phys. Rev. E, 2010, 82, 041128; doi 10.1103/PhysRevE.82.041128

23. Latorre J.I., Morris T.R., J. High Energy Phys., 2000, 11, 004; doi 10.1088/1126-6708/2000/11/004

24. Morris T.R., Phys. Lett. B, 1994, 334, 355; doi 10.1016/0370-2693(94)90767-6

25. Morris T.R., Int. J. Mod. Phys. A, 1994, 9, 2411; doi 10.1142/S0217751X94000972

26. Morris T.R., Nucl. Phys. B, 1996, 458, 477; doi 10.1016/0550-3213(95)00541-2

27. Sumi J.-I., Souma W., Aoki K.-I., Terao H., Morikawa K., Preprint $\operatorname{arXiv:hep-th/0002231,~} 2000$.

28. Morris T.R., Phys. Lett. B, 1994, 329, 241; doi 10.1016/0370-2693(94)90767-6

29. Morris T.R., Prog. Theor. Phys. Suppl., 1998, 131, 395; doi 10.1143/PTPS.131.395

30. Ellwanger U., Z. Phys. C, 1994, 62, 503; doi 10.1007/BF01555911

31. Bervillier C., Phys. Lett. A, 2004, 332, 93; doi 10.1016/j.physleta.2004.09.037

32. Rosten O.J., J. Phys. A: Math. Theor., 2011, 44, 195401; doi 10.1088/1751-8113/44/19/195401

33. Osborn H., Twigg D. E., Ann. Phys., 2012, 327, 29; doi 10.1016/j.aop.2011.10.011

34. Rosten O.J., Preprint arXiv:1106.2544 2011.

35. Polchinski J., Nucl. Phys. B, 1984, 231, 269; doi 10.1016/0550-3213(84)90287-6

36. Ball R.D., Haagensen P.E., Latorre J.I., Moreno E., Phys. Lett. B, 1995, 347, 80; doi 10.1016/0370-2693(95)00025-G

37. O’Dwyer J.P., Osborn H., Ann. Phys., 2008, 323, 1859; doi 10.1016/j.aop.2007.10.005

38. Riedel E.K., Golner G.R., Newman K.E., Ann. Phys., 1985, 161, 178; doi 10.1016/0003-4916(85)90341-0

39. Bervillier C. (unpublished).

40. Golner G.R., Preprint arXiv:hep-th/9801124 1998.

41. Bell T.L., Wilson K.G., Phys. Rev. B, 1975, 11, 3431; doi 10.1103/PhysRevB.11.3431

\title{
Рівняння точної ренормалізаційної групи Вільсона і параметр аномальної вимірності
}

\author{
К. Бервільє \\ Лабораторія математики і теоретичної фізики, UMR 7350 (CNRS), Університет Франсуа Рабле, \\ 37200 Тур, Франція
}

Параметр аномальної вимірності, введений в нелінійний спосіб у першій історичній версії рівняння точної ренормалізаційної групи, порівнюється з сучасною методикою. Простий вираз для точно граничного (маргінального) надлишкового оператора слідує з цієї нелінійності, тоді як для того, щоб отримати цей результат у лінійному випадку необхідно розв'язати диференціальні рівняння першого порядку. Висвітлено роль цього оператора в побудові рівняння потоку.

Ключові слова: рівняння точної ренормалізаційної групи, аномальна вимірність 